\title{
World impact of kernel European Union 9 countries from Google matrix analysis of the world trade network
}

\author{
Justin Loye ${ }^{1,2}$, Leonardo Ermann ${ }^{3}$ and Dima L. Shepelyansky ${ }^{*^{*}}$ (1)
}

\author{
${ }^{*}$ Correspondence: \\ dima@irsamc.ups-tlse.fr \\ ${ }^{1}$ Laboratoire de Physique \\ Théorique, Université \\ de Toulouse, CNRS, UPS, \\ 31062 Toulouse, France \\ Full list of author information \\ is available at the end of the \\ article
}

\begin{abstract}
We use the United Nations COMTRADE database for analysis of the multiproduct world trade network. With this data, considered for years 2012-2018, we determined the world trade impact of the Kernel of EU 9 countries (KEU9), being Austria, Belgium, France, Germany, Italy, Luxembourg, Netherlands, Portugal, Spain, considered as one united country. We apply the advanced Google matrix analysis for investigation of the influence of KEU9 and show that KEU9 takes the top trade network rank positions thus becoming the main player of the world trade being ahead of USA and China. Our network analysis provides additional mathematical grounds in favor of the recent proposal (Saint-Etienne in: Osons I'Europe des Nations. Editions de I'Observatoire/ Humensis, Paris, 2018) of KEU9 super-union which is based only on historical, political and economy basis.
\end{abstract}

Keywords: Complex networks, World trade, Google matrix, PageRank

\section{Introduction}

The economy of European Union (EU) is considered as the second world largest economy after United States (US) (2020) even if there are also other opinions placing China $(\mathrm{CN})$ on the first position (Largest Economies in the World 2020). At present EU includes 27 member states and about 447 million population (Wikipedia: European Union 2020). While the global EU economy and population are really huge the political action of member states (Largest Economies in the World 2020) is not always coherent pushing in some cases in different directions. Due to this reason there is a proposal, pushed forward by Christian Saint-Etienne, to consider the Kernel EU 9 (KEU9) states (or countries), which are tightly linked by historical, political and economic relations, as a strongly united kernel group of EU that would allow to perform coherent actions of these KEU9 states (Saint-Etienne 2018) (follow also discussion of this proposal at CNEWS 2019). These 9 kernel states include Austria (AT), Belgium (BE), France (FR), Germany (DE), Italy (IT), Luxembourg (LU), Netherlands (NL), Portugal (PT), Spain (ES) (Saint-Etienne 2018) with the total population of about 305 millions (Wikipedia: European Union 2020).

(c) The Author(s), 2021. Open Access This article is licensed under a Creative Commons Attribution 4.0 International License, which permits use, sharing, adaptation, distribution and reproduction in any medium or format, as long as you give appropriate credit to the original author(s) and the source, provide a link to the Creative Commons licence, and indicate if changes were made. The images or other third party material in this article are included in the article's Creative Commons licence, unless indicated otherwise in a credit line to the material. If material is not included in the article's Creative Commons licence and your intended use is not permitted by statutory regulation or exceeds the permitted use, you will need to obtain permission directly from the copyright holder. To view a copy of this licence, visit http:// creativecommons.org/licenses/by/4.0/. 
A variety of arguments in favor of possible coherent political and economic actions of KEU9 group is presented and analyzed in (Saint-Etienne 2018). However, this analysis is not based on detailed mathematical grounds pushing forward arguments of historical, political and economic heuristic reasons. Here, we put forward the mathematical foundations for this KEU9 proposal presenting the mathematical and statistical analysis of the world trade database of UN COMTRADE (2020). This database presents an exceptional variety of data on trade exchange between all world UN registered countries on a scale of more than 50 years with more than $10^{4}$ of trade commodities (products). The transactions are expressed in their dollar (USD) values of a given year. The World Trade Organization (WTO) Statistical Review (2018) demonstrates the vital importance of the international trade between countries for their development and progress. Also the whole world economy is deeply influenced by the world trade (Krugman et al. 2011). Hence, this database is well appropriate for verification of how strong and important is the world influence of KEU9 group on the world economy. Thus here we use the UN COMTRADE database (2020) for mathematical and statistical analysis of heuristic arguments presented in favor of KEU9 in Saint-Etienne (2018).

The trade transfer between countries represents the multiproduct World Trade Network (WTN). The modern methods of Google matrix approach (Brin and Page 1998; Langville and Meyer 2006; Ermann et al. 2015) are well suited for the analysis of transactions on the WTN. The detailed description of Google matrix applications to WTN are described in Ermann and Shepelyansky (2011, 2015), Coquidé et al. $(2019,2020)$. Here we apply these methods considering KEU9 countries as one country thus excluding trade transfers between them and keeping only ingoing and outgoing trade flows to this group from other countries.

We point that various research groups investigated the statistical properties of WTN (see e.g. Serrano et al. 2007; Fagiolo et al. 2009; He and Deem 2010; Fagiolo et al. 2010; Barigozzi et al. 2010; Benedictis and Tajoli 2011; Deguchi et al. 2014). However, as discussed in Ermann and Shepelyansky (2011, 2015), Coquidé et al. (2019) the Google matrix approach has significant advantages for analysis of weighted directed trade networks since it takes into account multiple iterative transactions and thus provides a new and more detailed analysis of trade influence propagation compared to the usual approach based on export and import flows.

\section{Materials and methods}

\section{Google matrix construction of WTN}

We consider the trade exchange between $N_{c}=186$ (185 countries + KEU9) world countries and $N_{p}=10$ products given by 1 digit from the the Standard International Trade Classification (SITC) Rev. 1, and for years 2012, 2014, 2016, 2018 taken from UN COMTRADE (2020). These 10 products contain all smaller subdivided specific products which number goes up to $10^{4}$. The list of these 10 products is given in Table 1 . The list of world countries is available at Ermann and Shepelyansky $(2011,2015)$. Following the approach developed in Ermann and Shepelyansky $(2011,2015)$ we obtain $N_{p}$ money matrices $M_{c, c^{\prime}}^{p}$ which give product $p$ transfer (in USD) from country $c^{\prime}$ to country $c$. The Google matrices $G$ for the direct trade flow and $G^{*}$ for the inverted trade flow have the size of $N=N_{c} N_{p}=1860$ nodes. They are constructed by normalization of all column 
Table 1 Product code and name for SITC classification level 1

\begin{tabular}{ll}
\hline Code & Name \\
\hline 0 & Food and live animals \\
1 & Beverages and tobacco \\
2 & Crude materials,inedible,except fuels \\
3 & Mineral fuels etc \\
4 & Animal and vegetable oils and fats \\
5 & Chemicals and related products,n.e.s. \\
6 & Basic manufactures \\
7 & Machinery,transport equipment \\
8 & Miscellaneous manufactured articles \\
9 & Goods not classified elsewhere \\
\hline
\end{tabular}

of outgoing weighted links to unity. There is also the part with a damping factor $\alpha=0.5$ describing random trade-surfer jumps to all nodes with a certain personalized vector taking into account the weight of each product in the global trade volume. The construction procedure of $G$ and $G^{*}$ is described in detail in Ermann and Shepelyansky (2015), Coquidé et al. (2019). The general properties and various examples of Google matrices of various networks are given in Brin and Page (1998), Langville and Meyer (2006), Ermann et al. (2015).

The stationary probability distribution of Markov transitions described by the Google matrix $G$ is given by the PageRank vector $P$ with maximal eigenvalue $\lambda=1$ : $G P=\lambda P=P$ (Brin and Page 1998; Langville and Meyer 2006). For the inverted flow described by $G^{*}$ matrix we have similarly the CheiRank vector $P^{*}$, being the eigenvector of $G^{*} P^{*}=P^{*}$. The importance and detailed statistical analysis of the CheiRank vector were demonstrated in Chepelianskii (2010) (see also Ermann and Shepelyansky 2011, 2015; Zhirov et al. 2010). We define PageRank $K$ and CheiRank $K^{*}$ indexes by monotonic ordering of probabilities of PageRank vector $P$ and of CheiRank vector $P^{*}$ as $P(K) \geq P(K+1)$ and $P^{*}\left(K^{*}\right) \geq P^{*}\left(K^{*}+1\right)$ with $K, K^{*}=1, \ldots, N$. By taking a sum over all products $p$ we obtain the PageRank and CheiRank probabilities of a given country as $P_{c}=\sum_{p} P_{c p}$ and $P^{*}{ }_{c}=\sum_{p} P^{*}{ }_{c p}$ (and in a similar way product probabilities $P_{p}, P^{*}{ }_{p}$ ) (Ermann and Shepelyansky 2015; Coquidé et al. 2019). From these probabilities we obtain the related indexes $K_{c}, K^{*}{ }_{c}$. In a similar way we define from import and export trade volume the probabilities $\hat{P}_{p}, \hat{P}_{p}^{*}, \hat{P}_{c}, \hat{P}_{c}^{*}, \hat{P}_{p c}, \hat{P}_{p c}^{*}$ and corresponding indexes $\hat{K}_{p}, \hat{K}_{p}^{*}$, $\hat{K}_{c}, \hat{K}_{c}^{*}, \hat{K}, \hat{K}^{*}$ (the import and export probabilities are normalized to unity via the total import and export volumes, see details in Ermann and Shepelyansky (2015), Coquidé et al. (2019)). We note that qualitatively PageRank probability is proportional to the volume of ingoing trade flow and CheiRank respectively to outgoing flow. Thus, approximately we can say that the high import gives a high PageRank $P$ and a high export a high CheiRank $P^{*}$ probabilities.

\section{Reduced Google matrix}

We also use the REGOMAX algorithm described in detail in Frahm (2016), Frahm et al. (2016). This algorithm allows to compute efficiently areduced Google matrix $G_{R}$ 
of size $N_{r} \times N_{r}$ that accounts all transitions of direct and indirect pathways happening in the full Google matrix $G$ between $N_{r}$ nodes of interest. For the selected $N_{r}$ nodes their PageRank probabilities are the same as for the global network with $N$ nodes (up to a constant multiplicative factor which takes into account that the sum of PageRank probabilities over $N_{r}$ nodes is unity). The matrix $G_{R}$ can be presented as as a sum of three matrix components that clearly distinguish direct and indirect interactions: $G_{\mathrm{R}}=G_{r r}+G_{\mathrm{pr}}+G_{\mathrm{qr}}$ (Frahm et al. 2016). Thus $G_{r r}$ is produced by the direct links between selected $N_{r}$ nodes in the global network of $N \gg N_{r}$ nodes. The component $G_{p r}$ is rather close to the matrix in which each column is given by the PageRank vector $P_{r}$ (up to a constant multiplier). Due to that $G_{p r}$ does not give much information about direct and indirect links between selected $N_{r}$ nodes. The most interesting and nontrivial role is played by the component $G_{q r}$, which accumulates the contribution of all indirect links between selected $N_{r}$ nodes appearing due to multiple pathways via the global network of $N$ nodes. The exact formulas for these three components of $G_{R}$ are given in (Frahm 2016; Frahm et al. 2016).

\section{Sensitivity of trade balance}

Following Ermann and Shepelyansky (2015), Coquidé et al. (2019), we use the trade balance of a given country with PageRank and CheiRank probabilities defined as $B_{c}=\left(P_{c}^{*}-P_{c}\right) /\left(P_{c}^{*}+P_{c}\right)$. In a similar way we have from ImportRank and ExportRank probabilities as $\hat{B}_{c}=\left(\hat{P}_{c}^{*}-\hat{P}_{c}\right) /\left(\hat{P}_{c}^{*}+\hat{P}_{c}\right)$. The sensitivity of trade balance $B_{c}$ to the price of energy or machinery can be obtained from the change of corresponding money volume flow related to SITC Rev.1 code $p=3$ (mineral fuels) or $p=7$ (machinery) by multiplying it by $(1+\delta)$, then computing all rank probabilities and the derivative $d B_{c} / d \delta$.

The efficiency of the above Google matrix methods has been demonstrated not only for the WTN but also for variety of other directed networks including Wikipedia
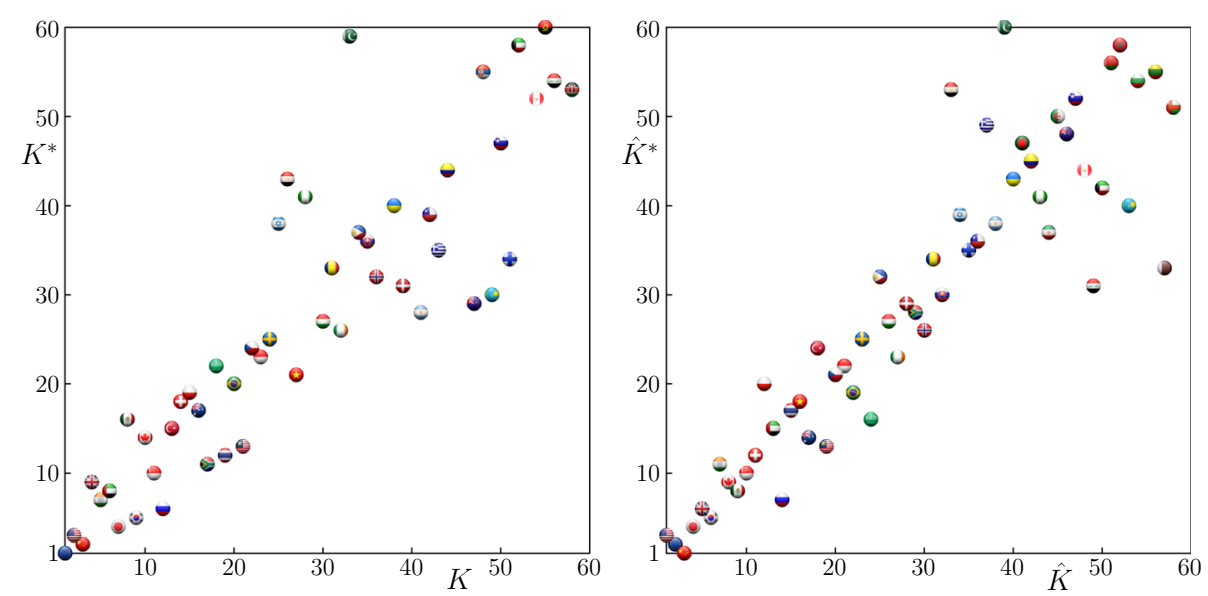

Fig. 1 PageRank-CheiRank plane of countries in 2018. Circles with country flags show positions of countries on the plane of PageRank-CheiRank indexes $\left(K, K^{*}\right)$ (summation is done over all products) (left panel) and on the plane of ImportRank-ExportRank $\hat{K}, \hat{K}^{*}$ from trade volume (right panel); data are shown only for index values less than 61 for year 2018; data for years 2012, 2014, 2016 are shown in Additional file 1: Fig. A1; KEU9 is marked by EU flag 
Table 2 Top 20 Ranking of PageRank $(K)$, CheiRank $\left(K^{*}\right)$, ImportRank and ExportRank for the year 2018

\begin{tabular}{lllll}
\hline Rank & PageRank $(K)$ & CheiRank $\left(K^{*}\right)$ & ImportRank & ExportRank \\
\hline 1 & KEU9 & KEU9 & USA & China \\
2 & USA & China & KEU9 & KEU9 \\
3 & China & USA & China & USA \\
4 & United Kingdom & Japan & Japan & Japan \\
5 & India & Republic of Korea & United Kingdom & Republic of Korea \\
6 & U. Arab Emirates & Russian Federation & Republic of Korea & United Kingdom \\
7 & Japan & India & India & Russian Federation \\
8 & Mexico & U. Arab Emirates & Canada & Mexico \\
9 & Republic of Korea & United Kingdom & Mexico & Canada \\
10 & Canada & Singapore & Singapore & Singapore \\
11 & Singapore & South Africa & Switzerland & India \\
12 & Russian Federation & Thailand & Poland & Switzerland \\
13 & Turkey & Malaysia & U. Arab Emirates & Malaysia \\
14 & Switzerland & Canada & Russian Federation & Australia \\
15 & Poland & Turkey & Thailand & U. Arab Emirates \\
16 & Australia & Mexico & Vietnam & Saudi Arabia \\
17 & South Africa & Australia & Australia & Thailand \\
18 & Saudi Arabia & Switzerland & Turkey & Vietnam \\
19 & Thailand & Poland & Malaysia & Brazil \\
20 & Brazil & Brazil & Czechia & Poland \\
\hline
\end{tabular}

The table lists country ranks of both panels of Fig. 1

networks (Zhirov et al. 2010; Zant et al. 2018; Demidov et al. 2020) and biological networks of protein-protein interactions (Lages et al. 2018; Zinovyev et al. 2020; Frahm and Shepelyansky 2020).

\section{Results}

\section{CheiRank and PageRank of countries}

We start the presentation of obtained results from showing the distribution of world countries on the plane of CheiRank-PageRank indexes $\left(K, K^{*}\right)$ given in Fig. 1 (left panel). Here, for a better visibility, we show only countries with $K, K^{*} \leq 60$, each country is marked by a circle with its flag. For a comparison we also present in Fig. 1 (right panel) the distribution of countries on the plane of ExportRank-ImportRank $\hat{K}, \hat{K}^{*}$ (in both panels, for compactness, we keep index $K$ which in fact corresponds to $K_{c}$ index of a country obtained by a summation over all products). The top 20 countries with their indexes are given in Table 2.

The main feature of Fig. 1 and Table 2 is that KEU9 takes the top leading position in PageRank and CheiRank indexes $K, K^{*}$ in 2018 (this leadership is also present in other studied years 2012, 2014, 206 as it is shown in Additional file 1: Fig. A1). This result is significantly different from the Import-Export volume ranking where in 2018 China is leading in export and USA in import. We argue that the Google matrix analysis via PageRank and CheiRank treats in a deeper way the multiplicity of trade relations between 
world countries compared to the standard Import-Export approach which takes into account only one step trade links.

Another important feature of Google matrix analysis is a significant improvement of positions of certain countries compared to their usual Import-Export ranking (see Fig. 1, Table 2). Thus Russia moves to the fourth CheiRank position $K^{*}=6$ compared to its ExportRank $\hat{K}^{*}=7$. Also India has strong CheiRank-PageRank position $K^{*}=7, K=5$ compared to Export-ImportRanks $\hat{K}^{*}=11, \hat{K}=7$. Also there is a significant reduction of positions of Switzerland from $\hat{K}^{*}=12, \hat{K}=11$ to $K^{*}=18, K=14$. In our opinion these results demonstrate a significant hidden power or weakness of trade relations of certain countries due to the multiplicity and variety of their trade relations which are not visible in a standard Export-Import approach.

We also considered the WTN rank positions for other unions of countries instead of KEU9 including countries of Shengen; European Economic Area; post Brexit EU of 27 countries (EU27 without UK). For all these cases we obtained the same PageRank and CheiRank positions as in Table 2for USA, China and the above union replacing KEU9. The main message of the results of this part is the world top leading position of KEU9 in CheiRank and PageRank trade that gives confirmation of the strength and importance

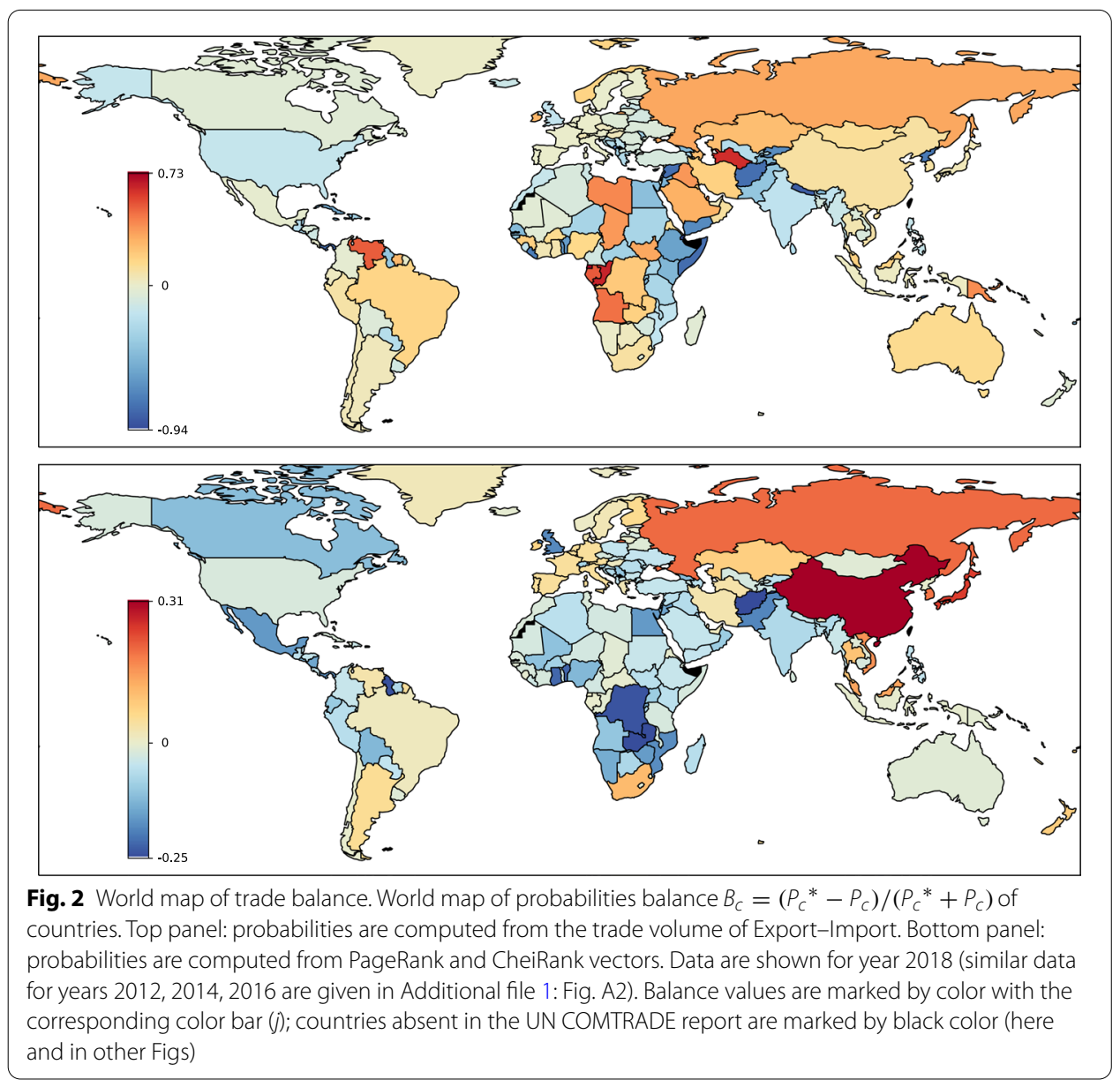


of KEU9 countries discussed in Saint-Etienne (2018). The detailed analysis of EU27 case will be presented elsewhere.

\section{Trade balance of countries}

We present the world map of trade balance $B_{c}$ of countries obtained from CheiRankPageRank and ExportRank-ImportRank probabilities in Fig. 2 for year 2018 (other years 2012, 2014, 2016 are given in Additional file 1: Fig. A2; the distributions of import and export of countries for all years are shown in Additional file 1: Figs. A3, A4).

The comparison of two ways of balance computation shows that Export-Import approach does not capture the influence of Russia and China on the world trade exchange. In contrast the CheiRank-PageRank approach directly highlights the multistep network influence of Russia and China on the world trade flows and their balance. We also see a strong positive CheiRank-PageRank balance for Japan. In both approaches the balance of US is close slightly negative. There is a relative increase of KEU9 balance in CheiRank-PageRank description compared to the standard Export-Import one. We attribute this to the fact that CheiRank-PageRank description takes into account the multiplicity of trade links which better describes a broad variety of KEU9 trade exchange.

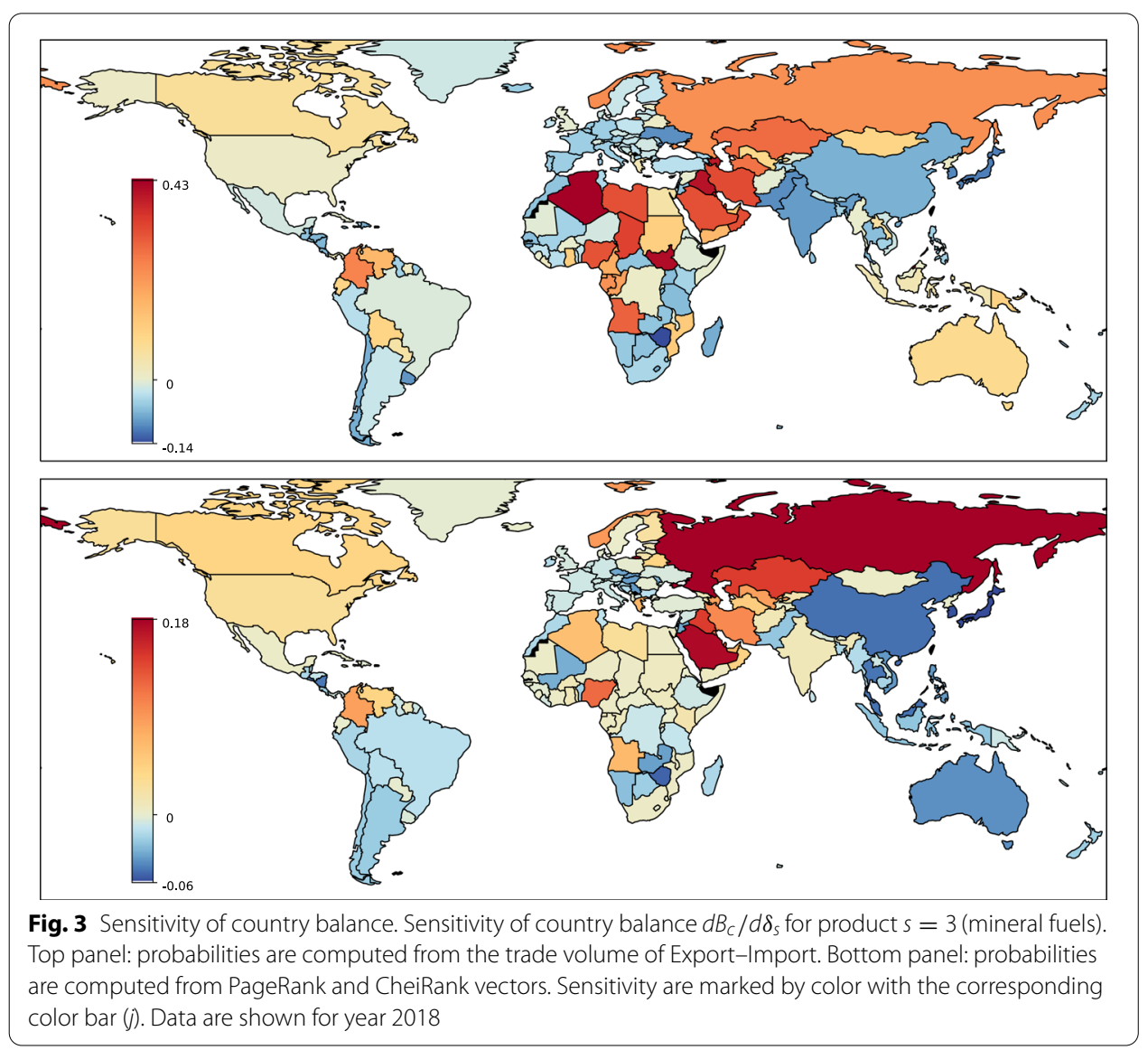




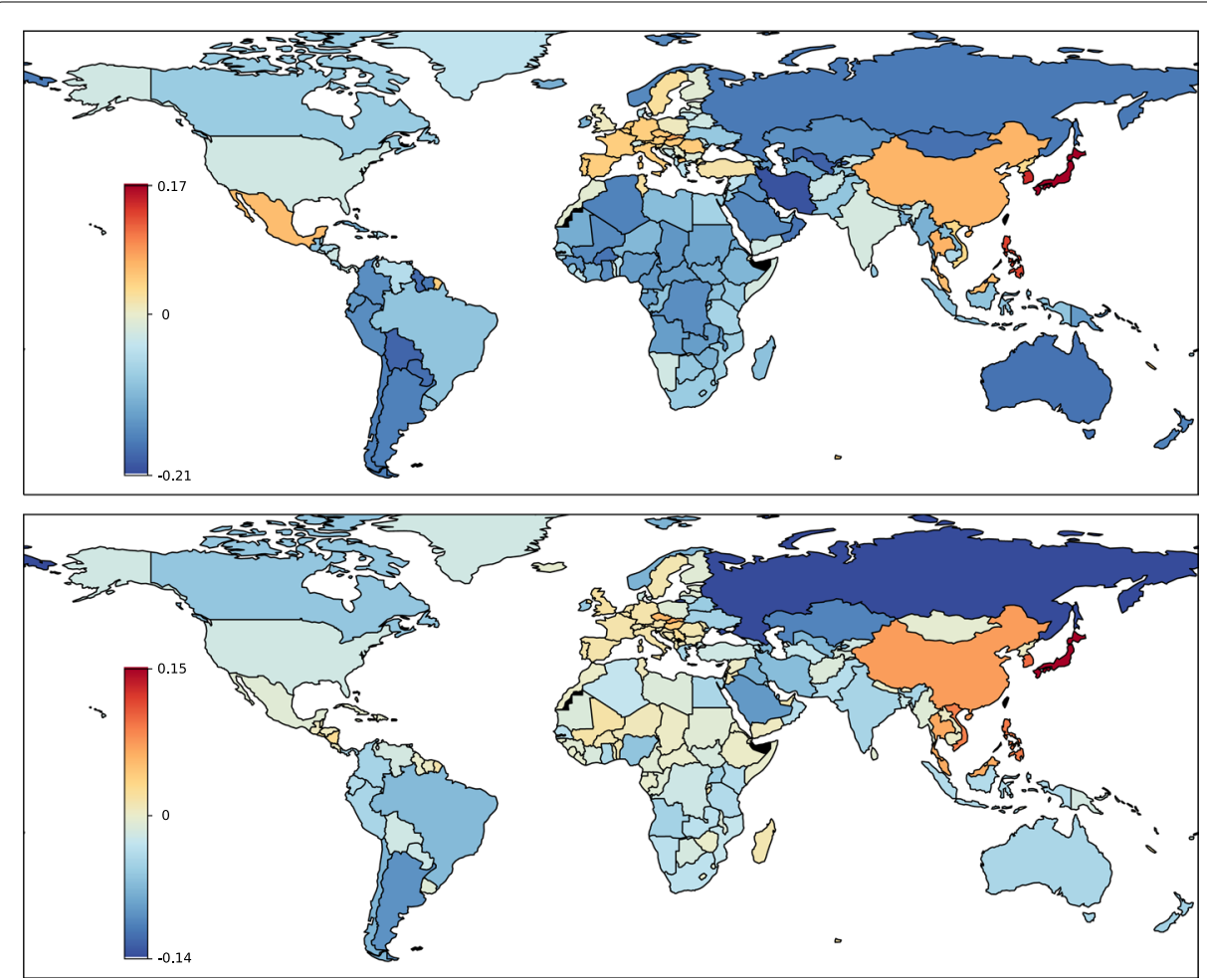

Fig. 4 Sensitivity of country balance. Same as in Fig. 3 but for product $s=7$ (machinery). Data are shown for year 2018 (similar data for years 2012, 2014, 2016 are given in Additional file 1: Fig. A6)

\section{Sensitivity of trade balance to specific products}

As described above we determine the sensitivity of trade balance of countries $d B_{c} / d \delta_{s}$ to specific products using the sensitivity definition from CheiRank-PageRank and ExportImport probabilities. The sensitivity results for $s=3$ (mineral fuels) are given in Fig. 3 . The CheiRank-PageRank approach shows that the most profitable countries with the highest values of $d B_{c} / d \delta_{3}$ are Saudi Arabia and Russia (Kazakhstan also has high sensitivity). This is rather natural since these countries are the highest petroleum producers. The strongly negative impact is well visible for Australia, China and countries of Latin America. USA and KEU9 sensitivities being close to zero.

In contrast the sensitivity from Export-Import approach gives of the top position Algeria (followed by Brunei). Among countries with strongly negative sensitivities we have India, Pakistan and China while Australia is slightly positive. In this Export-Import approach USA is slightly positive and KEU9 is slightly negative.

This shows a significant difference between the usual Export-Import analysis and the Google matrix approach. We argue that the latter approach takes into account the multiplicity of trade links and flows thus highlighting in a better way the multistep trade relations between countries.

The sensitivities of countries to the product $s=7$ (machinery) is shown in Fig. 4 . Here both approaches give the most positive countries being Japan, S.Korea and China. In the Export-Import approach KEU9 has a bit higher positive sensitivity 

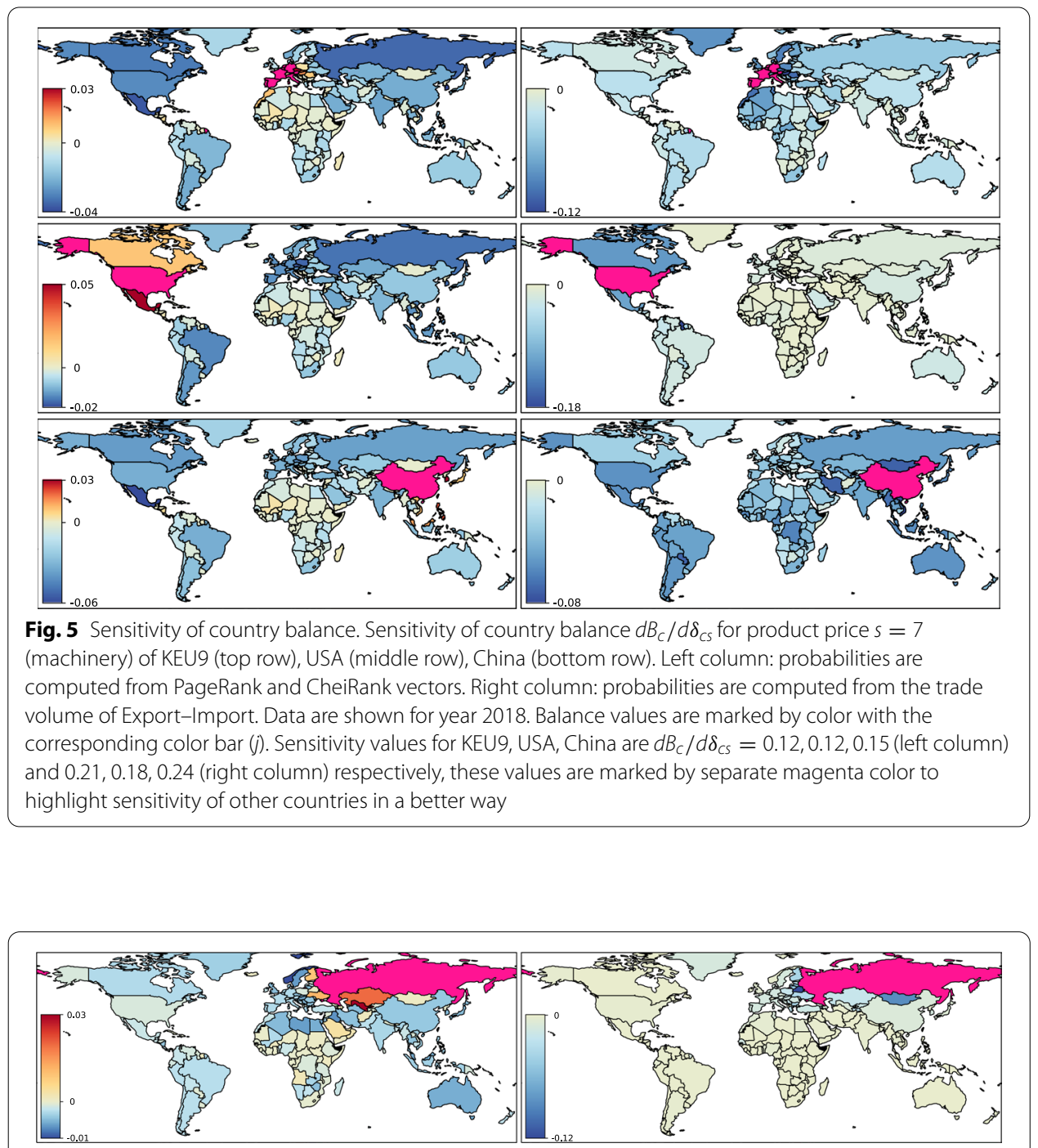

Fig. 6 Sensitivity of country balance. Sensitivity of country balance $d B_{c} / d \delta_{c S}$ for product price $s=3$ (mineral fuels) of Russia. Left panel: probabilities are computed from PageRank and CheiRank vectors; Right panel: probabilities are computed from the trade volume of Export-Import. Data are shown for year 2018. Sensitivity values for Russia are $d B_{c} / d \delta_{c s}=0.13$ (left panel) and 0.24 (right panel); these values are marked by separate magenta color to highlight sensitivity of other countries in a better way

compared to the CheiRank-PageRank method. Thus we have for both methods of CheiRank-PageRank and Export-Import: KEU9 $d B_{c} / d \delta_{7}=0.015, d \hat{B}_{c} / d \delta_{7}=0.043$; slightly negative values for USA $d B_{c} / d \delta_{7}=-0.019, d \hat{B}_{c} / d \delta_{7}=-0.027$; Russia has strongly negative values $d B_{c} / d \delta_{7}=-0.145, d \hat{B}_{c} / d \delta_{7}=-0.169$.

In the above Figs. 3, 4 we presented results for year 2018. The same type of data for years 2012, 2014, 2016 are given in Additional file 1: Figs. A5, A6.

Above we considered the sensitivity of trade balance to a global price variation of a given product applicable to the whole world with a homogeneous price increase of product for all countries. It is also interesting to consider the sensitivity of country trade balance when the product price is changed only by one country. In this way 
we obtain the sensitivity $d B_{c} / d \delta_{c s}$ of countries to a product of a given country. This specific sensitivity is shown in Fig. 5 in respect to price variation of $s=7$ (machinery) from KEU9, USA, and China in year 2018. The Export-Import approach gives strongly positive sensitivity for the country which increased price of machinery (respectively KEU9, USA, China). All other countries have sensitivity close to zero or negative. The result from CheiRank-PageRank analysis is different. For KEU9 machinery price increase the positive sensitivity is obtained for Czechia, Slovakia, Hungary (with the sensitivity values $0.028,0.017,0.015$ respectively). For USA case the positive sensitivity is obtained for Mexico, Canada with repective values $0.045,0.014$. For China case the positive sensitivity is obtained for Korea, Philippines, Malaysia (with sensitivity respective values $0.031,0.023,0.014$ ). This increase is related to strong network links between these countries well captured by the Google matrix analysis.

In Fig. 6 we show the sensitivity $d B_{c} / d \delta_{c s}$ from both approaches for $s=3$ (mineral fuels) of Russia. Again as for $s=7$ we see that the Export-Import approach gives the strong positive sensitivity only for Russia. In contrast the CheiRankPageRank approach shows that Uzbekistan, Kazakhstan, Ukraine (with values $d B_{c} / d \delta_{c s}=0.032,0.021,0.012$ respectively) also gain the positive sensitivity in the case of price increase of $s=3$ of Russia. This also confirms the strength of Google matrix analysis which captures multiple trade links between countries.

\section{Sensitivity of trade balance to labor cost}

It is interesting to analyze the sensitivity of a country trade balance $d B_{c} / d \sigma_{c^{\prime}}$ to a labor cost variation in a given country. This analysis is done by increasing the price of all products of a given country by a factor $1+\sigma_{c}$ followed by a renormalization of sum all column elements to unity. Such an approach has been developed and studied in Kandiah et al. (2015) for the world economic activities from World Trade Organization data. Here, at the difference of price shock of one product, the price increase affects all product flows from a given country corresponding to a global increase of

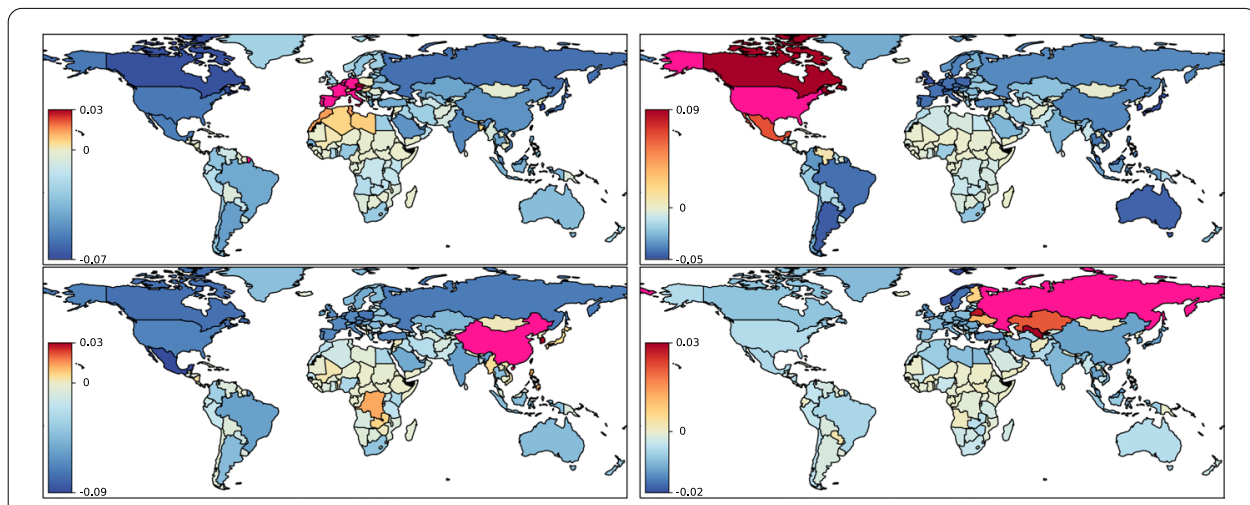

Fig. 7 Labor cost sensitivity. Labor cost sensitivity of country balance $d B_{c} / d \sigma_{c^{\prime}}$ for KEU9 (top left panel), USA (top right panel), China (bottom left panel), Russia (bottom right panel) for year 2018 (sensitivity is obtained from PageRank-CheiRank probabilities). The diagonal term $d B_{c} / d \sigma_{c}$ is shown by a fixed magenta color with numerical values being $d B_{c} / d \sigma_{c}=0.30,0.31,0.30,0.29$ respectively for KEU9, USA, China, Russia (this allows to highlight the effect for other countries in a better way) 
labor cost in a given country. Of course, the price increase is considered to be very small corresponding to the linear response regime. The labor cost sensitivity $d B_{c} / d \sigma_{c^{\prime}}$ is computed numerically in the same manner as the product sensitivity $d B_{c} / d \delta_{c^{\prime}}$ discussed above.

As discussed in Kandiah et al. (2015) the most strong labor cost sensitivity $d B_{c} / d \delta_{c^{\prime}}$ is naturally obtained for the country itself with $c=c^{\prime}$. Therefore, below in Fig. 7 we present the diagonal results for $d B_{c} / d \delta_{c^{\prime}}$ at $c \neq c^{\prime}$ by a separate magenta color while all other countries sensitivity are characterized by color bar.

For KEU9 the strongest sensitivity values $d B_{c} / d \delta_{c^{\prime}}$ are obtained for countries: Czechia, Tunisia, Morocco with positive values 0.027, 0.014, 0.013 and S.Korea, Canada, Russia with negative values $-0.074,-0.072,-0.062$. For USA case these are: Canada, Mexico, Venezuela with positive values 0.086, 0.063, 0.008 and S.Korea, Japan, United Kingdom with negative values $-0.045,-0.044,-0.044$; for China we

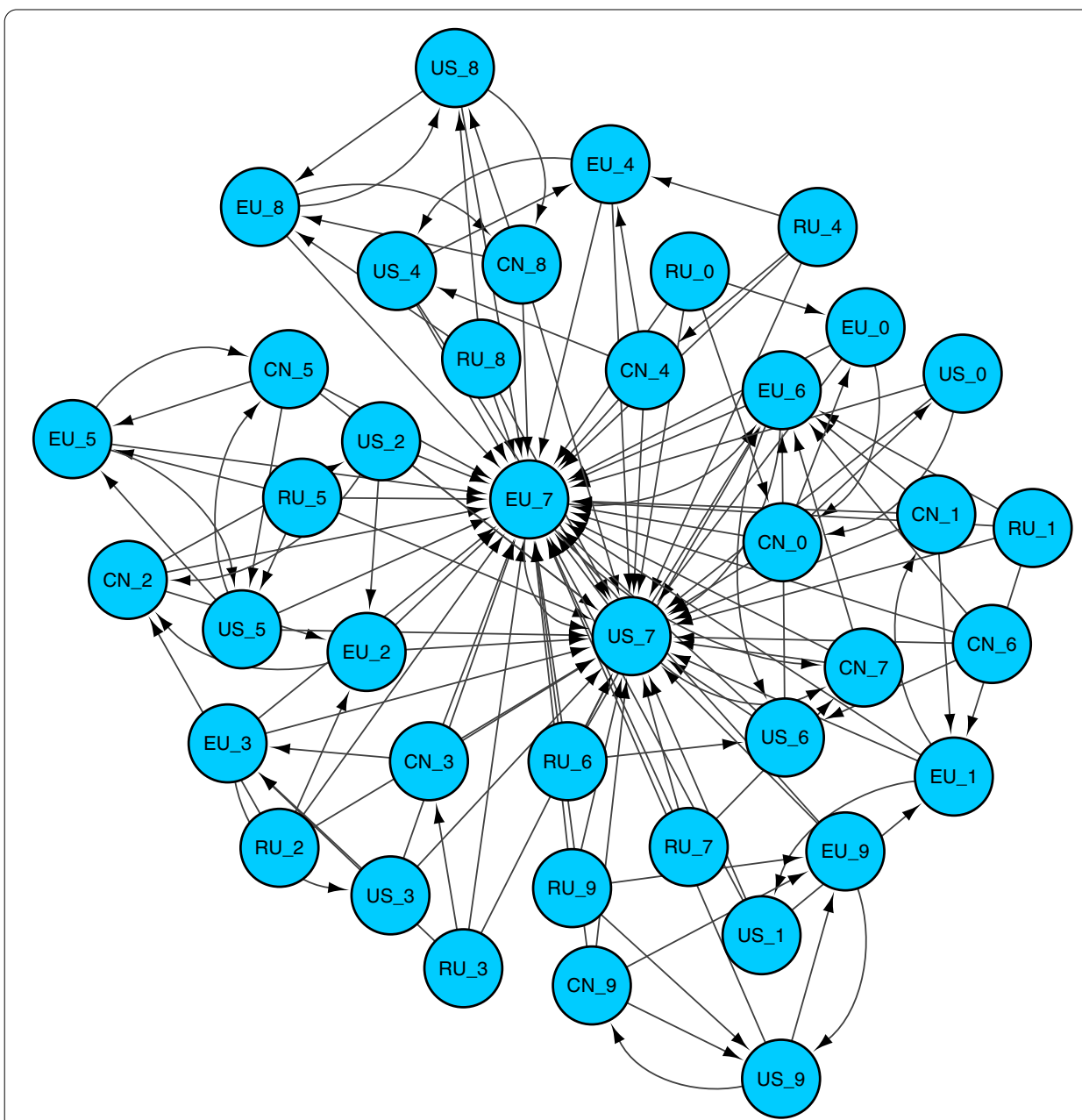

Fig. 8 Network trade structure. Network trade structure between KEU9 (marked as EU), USA (US), China (CN), Russia (RU) with 10 products of Table 1 in year 2018. Network is obtained from the reduced Google matrix $G_{R}$ by tracing four strongest outgoing links. Countries are shown by circles with two letters of country and product index from Table 1. The arrow direction from node $A$ to node $B$ means that $B$ imports from $A$. All 40 nodes are shown 


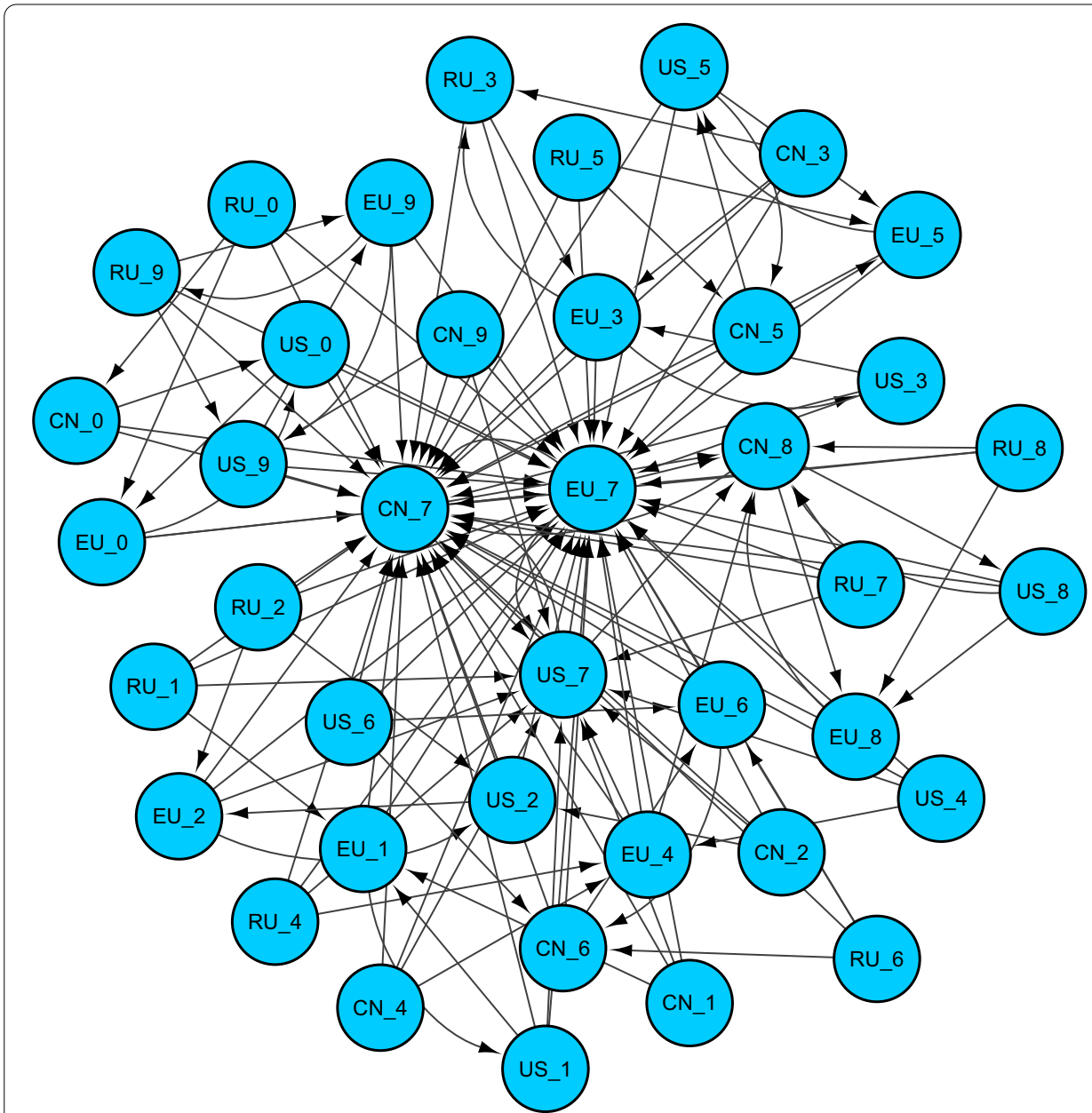

Fig. 9 Network trade structure. Same in Fig. 8 but for the reduced Google matrix $G^{*}{ }_{R}$ corresponding of inverted trade flows (CheiRank or Export direction). The arrow direction from node $A$ to node $B$ means that $B$ exports to $A$

find: Korea, Congo, Philippines with positive values 0.033, 0.015, 0.014 and Mexico, Canada, Poland with negative values $-0.090,-0.076,-0.074$. For Russia we obtain: Uzbekistan, Belarus, Kazakhstan with positive values 0.028, 0.026, 0.020 and Norway, Sweden, United Kingdom with negative values $-0.019,-0.016,-0.013$.

This shows that the Google matrix analysis allows to determine on pure mathematical grounds the mutual trade dependences of world countries.

\section{Network structure of trade from reduced Google matrix}

We use the REGOMAX algorithm described above to obtain the reduced Google matrix of trade flows between certain selected countries. We choose the case of 4 countries which has a strong world trade influence KEU9, USA, China, Russia with 10 trade products of Table 1. In this way the size of $G_{R}$ (Import or PageRank direct flow direction) and $G^{*}{ }_{R}$ (Export or CheiRank inverted flow direction) is equal to 40 . For clarity we show only 4 directed links corresponding to the most strong matrix elements from a given node 
(only non-diagonal terms are shown). The obtained networks are shown in Figs. 8 and 9 respectively.

The network structure shown in Fig. 8 from $G_{R}$ shows that the main importing nodes are machinery $(s=7)$ of KEU9 and USA. In a similar way the main exporting nodes of $G^{*}{ }_{R}$ are again machinery product of KEU9, China and USA (Fig. 9). This clearly show the importance of machinery product for the world trade.

\section{Discussion}

Above we considered the trade influence of kernel EU 9 countries (KEU9) considered as a one united state following the proposal pushed forward by Christian Saint-Etienne in Saint-Etienne (2018). The analysis is done on the bases of multiproduct trade data provided by UN COMTRADE (2020). Our results are based on the advanced Google matrix analysis of multiproduct world trade network flows for years 2012-2018 between all world countries registered at UN. They clearly show that KEU9 takes the world leading position in PageRank and CheiRank probabilities being ahead of USA and China. This mathematical network analysis demonstrates that KEU9 becomes the main player in the international trade. This provides additional mathematical foundation for the historical, economical and political arguments presented in Saint-Etienne (2018) in the favor of coherent strong impact of KEU9 (if united) on the world development.

We show that the Google matrix analysis allows to obtained significantly deeper information about world trade comparing to the Import-Export analysis usually used in economy studies.

Finally we note that the described Google matrix analysis can be also applied to financial networks of interbank payments. In fact their size is not very large, being about of only 7000 nodes for the Fedwire Funds Service (Soramaki et al. 2007). Up to now the matrix methods are quite rarely used in the field of financial transactions even if it was shown that the Random Matrix Theory finds useful applications for financial and credit risk analysis (Bouchaud and Potters 2003; Munnix et al. 2014). Also the methods of statistical mechanics demonstrated their efficiency for analysis of market economies (Bardoscia et al. 2017). However, the flows considered in Bouchaud and Potters (2003), Munnix et al. (2014) are non-directional while the WTN typically describes directed flows that is also a case of financial flows. Thus we expect that the Google matrix approach used here should work rather efficiently also for financial transfer systems.

Abbreviations

WTN: World trade network; KEU9: kernel of European Union 9 countries; REGOMAX: Reduced Google matrix.

\section{Supplementary Information}

The online version contains supplementary material available at https://doi.org/10.1007/s41109-021-00380-9.

Additional file 1. Additional file 1 of Suplnfo contains additional figures discussed in the main text of the article.

Acknowledgements

We thank Katia Jaffres-Runser (INP ENSEEIHT Toulouse) for useful discussions. We thank UN COMTRADE for providing to us a friendly access to their database.

Authors' contributions

The authors contributed equally to this work. All authors read and approved the final manuscript. 


\section{Funding}

This research has been partially supported through the Grant NANOX No. ANR-17-EURE-0009 (Project MTDINA) in the frame of the Programme des Investissements d'Avenir, France and in part by APR 2019 call of University of Toulouse and by Region Occitanie (Project GolA).

\section{Declaration}

\section{Competing interests}

The authors declare that they have no competing interests.

\section{Author details}

${ }^{1}$ Laboratoire de Physique Théorique, Université de Toulouse, CNRS, UPS, 31062 Toulouse, France. ${ }^{2}$ Institut de Recherche en Informatique de Toulouse, Université de Toulouse, UPS, 31062 Toulouse, France. ${ }^{3}$ Departamento de Física Teórica,

GlyA, Comisión Nacional de Energía Atómica, Av. del Libertador 8250, 1429 Buenos Aires, Argentina.

Received: 18 December 2020 Accepted: 19 May 2021

Published online: 26 May 2021

\section{References}

Bardoscia M, Livan G, Marsili M (2017) Statistical mechanics of complex economies. J Stat Mech 2017:043401. https://doi. org/10.1088/1742-5468/aa6688

Barigozzi M, Fagiolo G, Garlaschelli D (2010) Multinetwork of international trade: a commodity-specific analysis. Phys Rev E 81:048104. https://doi.org/10.1103/PhysRevE.81.046104

Benedictis LD, Tajoli L (2011) The world trade network. World Econ 34(8):1417. https://doi.org/10.1111/j.1467-9701.2011. 01360.x

Bouchaud J-P, Potters M (2003) Theory of financial risk and derivative pricing. Cambridge University Press, Cambridge

Brin S, Page L (1998) The anatomy of a large-scale hypertextual web search engine. Comput Netw ISDN Syst 30(1):107117. https://doi.org/10.1016/S0169-7552(98)00110-X

Chepelianskii AD (2010) Towards physical laws for software architecture. arXiv:1002.5455

CNEWS: Face a Linfo Avec Eric Zemmour Saison 1, 14 Nov 2019 (Retrieved November, 2020). https://www.cnews.fr/emiss ion/2019-11-14/face-linfo-du-14112019-899420

Coquidé C, Ermann L, Lages J, Shepelyansky DL (2019) Influence of petroleum and gas trade on EU economies from the reduced google matrix analysis of un comtrade data. Eur Phys J B 92(8):171. https://doi.org/10.1140/epjb/ e2019-100132-6

Coquidé C, Lages J, Shepelyansky DL (2020) Crisis contagion in the world trade network. Appl Netw Sci 5:67. https://doi. org/10.1007/s41109-020-00304-z

Deguchi T, Takahashi K, Takayasu H, Takayasu M (2014) Hubs and authorities in the world trade network using a weighted hits algorithm. PLoS ONE 9(7):100338. https://doi.org/10.1371/journal.pone.0100338

Demidov D, Frahm KM, Shepelyansky DL (2020) What is the central bank of Wikipedia? Physica A 542:123199. https://doi. org/10.1016/.jphysa.2019.123199

Ermann L, Shepelyansky DL (2011) Google matrix of the world trade network. Acta Physica Polonica A 120:158

Ermann L, Shepelyansky DL (2015) Google matrix analysis of the multiproduct world trade network. Eur Phys J B 88(4):84. https://doi.org/10.1140/epjb/e2015-60047-0

Ermann L, Frahm KM, Shepelyansky DL (2015) Google matrix analysis of directed networks. Rev Mod Phys 87:1261. https://doi.org/10.1103/RevModPhys.87.1261

Fagiolo G, Rayes J, Schiavo S (2009) World-trade web: topological properties, dynamics, and evolution. Phys Rev E 79:036115. https://doi.org/10.1103/PhysRevE.79.036115

Fagiolo G, Rayes J, Schiavo S (2010) The evolution of the world trade web: a weighted-network analysis. J Evol Econ 20:479. https://doi.org/10.1007/s00191-009-0160-x

Frahm KM, Shepelyansky DL (2016) Reduced Google matrix. arXiv:1602.02394

Frahm KM, Shepelyansky DL (2020) Google matrix analysis of bi-functional signor network of protein-protein interactions. Physica A 559:125019. https://doi.org/10.1016/.jphysa.2020.125019

Frahm KM, Jaffres-Runser K, Shepelyansky DL (2016) Wikipedia mining of hidden links between political leaders. Eur Phys J B 89:269. https://doi.org/10.1140/epjb/e2016-70526-3

He J, Deem MW (2010) Structure and response in the world trade network. Phys Rev Lett 105:198701. https://doi.org/10. 1103/PhysRevLett.105.198701

Kandiah V, Escaith H, Shepelyansky DL (2015) Google matrix of the world network of economic activities. Eur Phys J B 88:186. https://doi.org/10.1140/epjb/e2015-60324-x

Krugman P, Obstfeld M, Melitz M (2011) International economics: theory \& policy. DescriptionPrentice Hall, Hoboken

Lages J, Shepelyansky DL, Zinovyev A (2018) Inferring hidden causal relations between pathway members using reduced google matrix of directed biological networks. PLoS ONE 13(1):0190812. https://doi.org/10.1371/journal.pone.01908 12

Langville A, Meyer C (2006) Google's PageRank and beyond: the science of search engine rankings. Princeton University Press, Princeton

Largest Economies in the World (Retrieved November, 2020). https://www.thebalance.com/world-s-largest-economy3306044

Munnix MC, Schaefer R, Guhr T (2014) A random matrix approach to credit risk. PLoS ONE 9(5):98030. https://doi.org/10. 1371/journal.pone.0098030

Saint-Etienne C (2018) Osons l'Europe des Nations. Editions de l'Observatoire/Humensis, Paris 
Serrano MA, Boguna M, Vespignani A (2007) Patterns of dominant flows in the world trade web. J Econ Interact Coord 2(2):111. https://doi.org/10.1007/s11403-007-0026-y

Soramaki K, Bech ML, Arnold J, Glass RJ, Beyeler WE (2007) The topology of interbank payment flows. Physica A 379:317. https://doi.org/10.1016/j.physa.2006.11.093

United Nations Commodity Trade Statistics Database (Retrieved November, 2020). http://comtrade.un.org/db/

Wikipedia: European Union (Retrieved November, 2020). https://en.wikipedia.org/wiki/European_Union

Wikipedia: Economy of the European Union (Retrieved November, 2020). https://en.wikipedia.org/wiki/Economy_of_ the_European_Union

World Trade Organization Statistical Review (2018) (Retrieved November, 2020). https://www.wto.org/english/res_e/ statis_e/wts2018_e/wts18_toc_e.htm

Zant SE, Jaffres-Runser K, Shepelyansky DL (2018) Capturing the influence of geopolitical ties from Wikipedia with reduced google matrix. PLoS ONE 13(8):0201397. https://doi.org/10.1371/journal.pone.0201397

Zhirov AO, Zhirov OV, Shepelyansky DL (2010) Two-dimensional ranking of Wikipedia articles. Eur Phys J B 77:523. https:// doi.org/10.1140/epjb/e2010-10500-7

Zinovyev A, Czerwinska U, Cantini L, Barillot E, Frahm KM, Shepelyansky DL (2020) Collective intelligence defines biological functions in Wikipedia as communities in the hidden protein connection network. PLoS Comput Biol 16(2):1007652. https://doi.org/10.1371/journal.pcbi.1007652

\section{Publisher's note}

Springer Nature remains neutral with regard to jurisdictional claims in published maps and institutional affiliations.

Submit your manuscript to a SpringerOpen ${ }^{\circ}$ journal and benefit from:

- Convenient online submission

- Rigorous peer review

- Open access: articles freely available online

- High visibility within the field

Retaining the copyright to your article

Submit your next manuscript at $\boldsymbol{\Delta}$ springeropen.com 